\title{
Minimally Invasive Surgery for Intraocular Lens Removal and Intrascleral Intraocular Lens Fixation with Trabeculectomy in a Patient with Dislocated Intraocular Lens and Elevated Intraocular Pressure
}

\author{
Yuko Mano Kei Mizobuchi Tomoyuki Watanabe Akira Watanabe \\ Tadashi Nakano \\ Department of Ophthalmology, The Jikei University School of Medicine, Tokyo, Japan
}

\author{
Keywords \\ Trabeculectomy · Intraocular lens fixation · Double-needle technique - Intraocular lens \\ dislocation Elevated intraocular pressure
}

\begin{abstract}
A 88-year-old female who was being treated for end-stage pseudoexfoliation syndrome was referred to our hospital for treatment of dislocated intraocular lens (IOL) and the elevated intraocular pressure (IOP) and in the right eye (RE). At the first visit to our hospital, best-corrected visual acuity (BCVA) was 0.2 in the RE and 0.02 in the left eye (LE). IOP was $47 \mathrm{~mm} \mathrm{Hg}$ in the RE and $21 \mathrm{~mm} \mathrm{Hg}$ in the LE. Slit-lamp examination showed no abnormalities in anterior segments and dislocated IOL in the RE. Fundus photograph showed optic disc pallor in both eyes. We performed the combined therapy of flanged intrascleral IOL fixation with the double-needle technique and trabeculectomy. Throughout the follow-up period, BCVA slightly improved from 0.2 to 0.4 in the RE. The angle of tilt of the $\mathrm{IOL}$ was $6.6,7.9$, and $8.7^{\circ}$ as measured by swept-source optical coherence tomography at 1, 4, and 6 months after the surgery, respectively. The IOP remained less than $10 \mathrm{~mm} \mathrm{Hg}$ without having to administer any other glaucoma medications. Furthermore, any complications associated with the surgery were not confirmed.
\end{abstract}




\section{Introduction}

Previous studies have reported the combination of transscleral intraocular lens (IOL) suture fixation and trabeculectomy to treat a dislocated IOL or subluxated crystalline lens accompanied by elevated intraocular pressure (IOP) [1,2]. Those studies also reported an overall success rate of about $70-80 \%$ in terms of IOP control $[1,2]$. On the other hand, management of elevated IOP is well known to be difficult in some cases with higher preoperative IOP or patients requiring a larger number of preoperative antiglaucoma medications [1]. Recently, various intrascleral IOL fixation methods have also been used instead of the transscleral IOL suture technique [3, 4]. Among such methods, the double-needle technique has been considered both simpler and safer than other intrascleral IOL fixation techniques [5]. However, few studies have reported using a combined method comprising flanged intrascleral IOL fixation with the double-needle technique and trabeculectomy for patients with dislocated IOL and elevated IOP. The purpose of this case presentation was to report details, with a particular focus on surgical technique, for a patient with dislocated IOL and elevated IOP who exhibited good clinical course after treatment using the combined therapy.

\section{Case Report}

An 88-year-old female was being treated for end-stage pseudoexfoliation syndrome using 5 different antiglaucoma eye drops. Goldmann perimetry (Haag-Streit, Bern, Switzerland) showed $20^{\circ}$ of central visual field in I-4e isopter with preserved peripheral visual field in the right eye (RE) and $20^{\circ}$ of absolute central scotoma with constricted peripheral visual field in the left eye (LE). During a previous hospital visit, a sudden elevation of the IOP was seen after slight dislocation of the IOL in the RE. She was referred to our hospital for treatment of the elevated IOP and dislocated IOL.

At the first visit to our hospital, best-corrected visual acuity was 0.2 in the RE and 0.02 in the LE. IOP was $47 \mathrm{~mm} \mathrm{Hg}$ in the RE and $21 \mathrm{~mm} \mathrm{Hg}$ in the LE. Slit-lamp examination showed no abnormalities in anterior segments and a dislocated IOL in the RE. Fundus photograph showed optic disc pallor in both eyes. We decided to use a combined therapy of flanged intrascleral IOL fixation with the double-needle technique [1] and trabeculectomy for dislocated IOL and elevated IOP taking into consideration of preoperative higher IOP and poor visual function. A 25-gauge pars plana vitrectomy was performed using a Constellation Vision System (Alcon Laboratories, Inc., Duluth, GA, USA) under retrobulbar anesthesia. We created the 3 ports at the 4, 7, and 10 o'clock positions and a small L-shaped incision at the 8 o'clock position (Fig. 1a), with the aim of preserving the upper conjunctiva for performing trabeculectomy. The dislocated IOL was extracted from the L-shaped incision $(3 \times 3 \mathrm{~mm})$ at the 8 o'clock position (Fig. 1b), with a 3-piece IOL then inserted into the anterior chamber through the same incision. The IOL was fixed through sclerotomies, made by a 30 -gauge needle at the 4 and 10 o'clock positions, respectively (Fig. 1c, d). Trabeculectomy was then performed as follows. A fornix-based conjunctival incision (approximately $5.0 \mathrm{~mm}$ ) was made along the limbus. A double scleral flap was created at the 1 o'clock position, consisting of a superficial scleral flap with one-half the thickness of the sclera $(2.5 \times 2.5 \mathrm{~mm})$ and a deep scleral flap with one-quarter the thickness of the sclera $(2.0 \times 2.5 \mathrm{~mm})$ (Fig. 1e). Small pieces of surgical sponges soaked in $0.04 \%$ mitomycin $\mathrm{C}$ were placed under the scleral flap and conjunctiva for $3 \mathrm{~min}$. After peripheral iridectomy was performed, the scleral flap and conjunctiva were sutured with 10-0 nylon (Fig. 1f). During the 6-month follow-up after the surgery, the patient underwent periodic ophthalmic examinations. Best-corrected visual acuity slightly improved from 0.2 to 0.4 in the RE. Angle of tilt of the IOL as measured by swept-source optical coherence

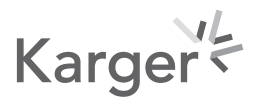


Mano et al.: Combined Surgical Technique of Intraocular Lens Fixation with Trabeculectomy

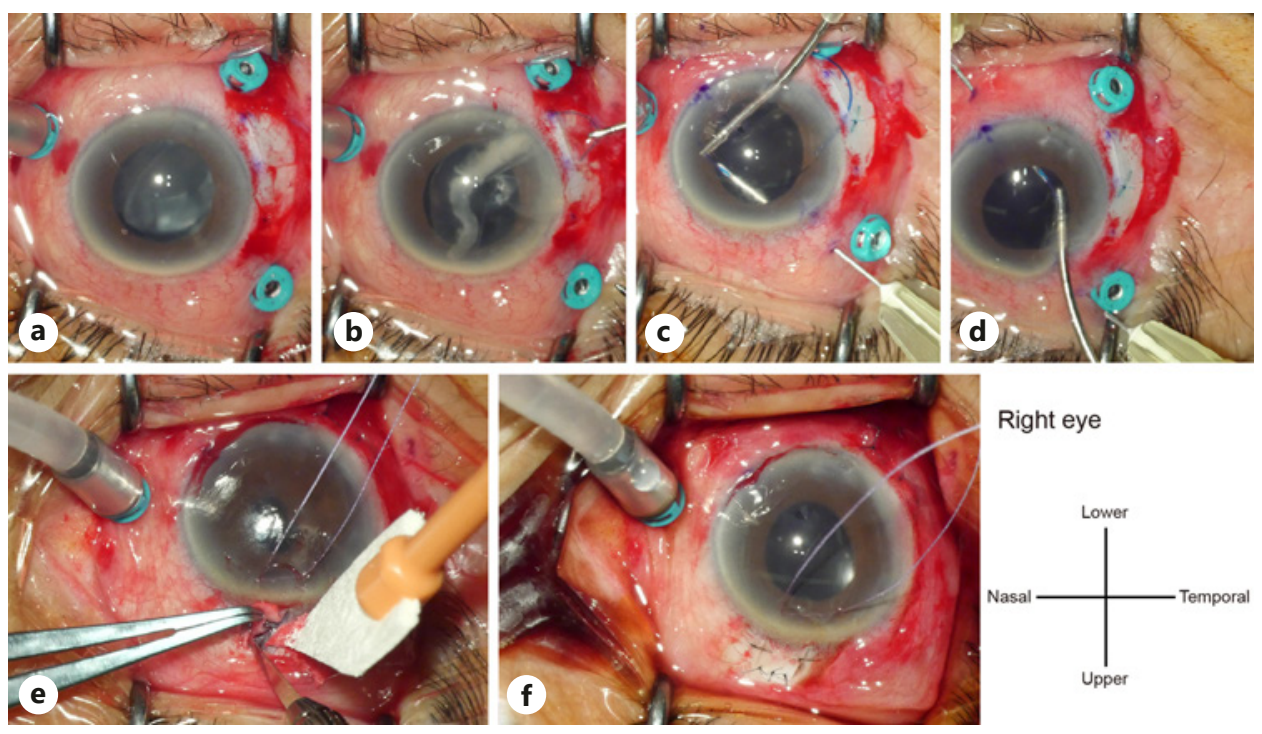

Fig. 1. Operative findings for the RE. a IOL is dislocated to inferior. Small incision wound of L shape for extraction of IOL is made at temporal (8 o'clock). b Dislocated IOL is extracted from the incision wound. After the extraction, a 3-piece IOL for the fixation is inserted into the incision wound. $\mathbf{c}, \mathbf{d}$ The IOL is fixed through sclerotomy, which is made by 30-gauge needle at 4 and 10 o'clock. e, $\mathbf{f}$ Trabeculectomy is performed at upper conjunctiva (1 o'clock). IOL, intraocular lens; RE, right eye.

tomography (SS-1000 CASIA; Tomey Corporation, Nagoya, Japan) was 6.6, 7.9, and $8.7^{\circ}$ at 1 , 4, and 6 months postoperatively, respectively. As for IOP, 2 of the 5 scleral sutures were cut by laser lysis for IOP control at 1 week after the surgery. The IOP was 10, 9, and $8 \mathrm{~mm} \mathrm{Hg}$ without having to administer any other glaucoma medications at 1, 4, and 6 months after the surgery, respectively. The filtration bleb remained in good condition without complications during the follow-up period.

\section{Discussion}

Previous studies have reported on cases of elevated IOP associated with a dislocated IOL or subluxated crystalline lens. In these previous cases, an overall success rate of about $70-80 \%$ was achieved in terms of IOP control $[1,2]$. However, elevated IOP management can be difficult in some cases. Shin et al. [1] reported that cases with a higher preoperative IOP and patients requiring a larger number of preoperative antiglaucoma medications tended to require additional surgical interventions such as trabeculectomy or glaucoma drainage devices [1]. David et al. [2] noted that the combination of lens fixation and trabeculectomy was particularly needed in cases with glaucomatous optic nerve damage in order to prevent a postoperative IOP spike. In the current patient, not only IOL fixation but also treatment for the elevated IOP was needed due to the risk of IOP remaining high and poor visual function in the other eye. The purpose of the current patient was to preserve the conjunctiva for creation of an adequate filtration bleb, resulting in lower IOP. During the surgery in the current patient, we extracted the dislocated IOL via a small lower temporal L-shaped incision, and a 3-piece IOL was then inserted into the anterior chamber at the same location because we wanted to preserve the upper conjunctiva for trabeculectomy. Furthermore, we chose to perform flanged intrascleral IOL fixation with the double-needle technique, which normally requires fewer conjunctival resections as compared to the transscleral IOL suture technique [5]. Our

\section{Karger's}


Mano et al.: Combined Surgical Technique of Intraocular Lens Fixation with Trabeculectomy

patient exhibited a good clinical course of adequate low IOP without glaucoma medications during follow-up period. When the results of the current and previous cases are considered together, we believe that the IOL fixation technique works well in combination with trabeculectomy, as more of the conjunctiva is preserved. However, 2 limitations need to be discussed when considering the results of the current patient. First, the follow-up period was relatively short. Second, the temporal conjunctiva needs to be preserved when extracting the dislocated IOL and positioning the 3-port. Further investigations will need to be undertaken to definitively clarify the ophthalmic outcomes in additional cases of dislocated IOL and elevated IOP that are treated using the combined therapy of flanged intrascleral IOL fixation with the double-needle technique and trabeculectomy.

In conclusion, we have reported a patient with dislocated IOL and elevated IOP who exhibited a good clinical course. The current results suggest that the use of combination therapy of flanged intrascleral IOL fixation with the double-needle technique and trabeculectomy might represent an effective procedure for patients with a dislocated IOL and elevated IOP.

\section{Acknowledgment}

We thank the patient for participating in this study.

\section{Statement of Ethics}

The protocol adhered to the tenets of the Declaration of Helsinki and informed consent was obtained from participants. The paper is exempt from ethical committee approval because our institute suggests single case report does not apply to that.

\section{Conflict of Interest Statement}

The authors declare no competing financial interest.

\section{Funding Sources}

This research did not receive any specific grant from funding agencies in the public, commercial, or not-for-profit sectors.

\section{Author Contributions}

Y.M., K.M., T.W., A.W., and T.N. contributed to the conception and design of this research. K.M. wrote the draft of this manuscript. T.W. contributed to the diagnosis and treatment of the patient. All the authors contributed to manuscript revision and approved the submitted final version.

\section{Karger'}




\section{References}

1 Shin DH, Birt CM, O'Grady JM, Kim C, Juzych MS, Lemon LC, et al. Transscleral suture fixation of posterior chamber lenses combined with trabeculectomy. Ophthalmology. 2001;108(5):919-29.

2 David RL, Balekudaru S, George RJ, Sen P, Lingam V. Management of elevated intraocular pressure associated with subluxated/dislocated lenses by combining trabeculectomy with adjunctive mitomycin C with lensectomy, vitrectomy, and scleral fixation of intraocular lens. J Glaucoma. 2016;25(7):e686-90.

3 Ohta T, Toshida H, Murakami A. Simplified and safe method of sutureless intrascleral posterior chamber intraocular lens fixation: Y-fixation technique. J Cataract Refract Surg. 2014;40(1):2-7.

4 Totan Y, Karadag R. Trocar-assisted sutureless intrascleral posterior chamber foldable intra-ocular lens fixation. Eye. 2012;26(6):788-91.

5 Yamane S, Sato S, Maruyama-Inoue M, Kadonosono K. Flanged intrascleral intraocular lens fixation with double-needle technique. Ophthalmology. 2017;124(8):1136-42. 